\title{
Complete histologic normalisation is associated with reduced risk of relapse among patients with ulcerative colitis in complete endoscopic remission
}

\author{
Kelly C. Cushing ${ }^{1,2,3}$ (D) | William Tan ${ }^{1,2}$ | David H. Alpers ${ }^{4}$ | Vikram Deshpande ${ }^{2,5}$ | \\ Ashwin N. Ananthakrishnan ${ }^{1,2}$
}

${ }^{1}$ Division of Gastroenterology,

Massachusetts General Hospital, Boston,

MA, USA

${ }^{2}$ Harvard Medical School, Boston, MA, USA

${ }^{3}$ Division of Gastroenterology, University of Michigan, Ann Arbor, MI, USA

${ }^{4}$ Division of Gastroenterology, Washington University School of Medicine, St. Louis, MO, USA

${ }^{5}$ Department of Pathology, Massachusetts General Hospital, Boston, MA, USA

\section{Correspondence}

Ashwin N. Ananthakrishnan, Massachusetts General Hospital Crohn's and Colitis Centre, 165 Cambridge Street, $9^{\text {th }}$ Floor, Boston, MA 02114, USA.

Email: aananthakrishnan@mgh.harvard.edu

\section{Funding information}

Ananthakrishnan is funded by the Crohn's and Colitis Foundation, National Institutes of Health (R03 DK112909) and the Chleck Family Foundation. This work is supported by the National Institutes of Health (P30 DK043351) to the Center for Study of Inflammatory Bowel Diseases.

\section{Summary}

Background: Clinical and endoscopic remission are treatment targets in ulcerative colitis (UC). The value of histologic healing in altering clinical outcomes among patients with complete endoscopic healing is not well established.

Aim: To quantify the association between histologic activity and clinical relapse among patients with UC who were in complete endoscopic remission.

Methods: This study included patients with UC from a prospective registry who were in complete endoscopic remission. Histologic activity was quantified by a senior gastrointestinal pathologist. Histologic activity was defined as lack of normalisation (Geboes score > 0) as well as histologically active disease (Geboes score $\geq 2.1$ and 23.1). The primary outcome was clinical relapse within 2 years. Multivariable regression adjusting for potential confounders examined the independent predictive value of histologic changes.

Results: The study included 83 patients (51\% women) (median age 44 years; median disease duration 11 years). Forty-one (49\%) had complete histologic normalisation. Within two years, 26 (31\%) experienced clinical relapse. Patients with complete histologic normalisation were less likely to experience relapse $(5 / 41,12 \%)$ compared to those without normalisation $(21 / 42,50 \%, P<0.001)$ (multivariable OR 7.22, 95\% confidence interval (CI) 2.48-24.70) by the Geboes score. The individual components of the Geboes score predictive of relapse were architectural changes $(P=0.03)$ and increased chronic inflammatory infiltrate $(P<0.001)$.

Conclusions: Complete histologic healing using the Geboes score was associated with reduced rates of clinical relapse among patients with UC in endoscopic remission. 


\section{1 | INTRODUCTION}

Ulcerative colitis (UC) is a chronic immune-mediated gastrointestinal disease that affects nearly 1 million Americans. ${ }^{1,2}$ Traditionally, the aim of medical therapy of UC has been relief from disease-related symptoms of rectal bleeding, urgency and diarrhoea. ${ }^{2-5}$ However, it is increasingly recognised that endoscopic resolution of inflammation is a more robust outcome. ${ }^{4-6}$ Endoscopic healing is associated with reduced need for corticosteroids, clinical relapse, risk of hospitalisation, surgery, as well as colorectal neoplasia. ${ }^{7-10}$ While the exact definition of endoscopic healing has varied, the treatment target most often recommended has been attainment of a Mayo endoscopic subscore of 0 or $1 .^{4,5}$ However, an emerging body of evidence has suggested that this definition may be too broad and that short-term and long-term outcomes are superior with a Mayo endoscopic score of 0 (completely normal mucosa) compared to a score of 1 , leading to the former being termed endoscopic remission and a score of 0 or 1 being termed endoscopic improvement. ${ }^{11,12}$ Furthermore, therapeutic intervention among those with a Mayo score of 1 has been associated with reduced risk of relapse, supporting a more stringent endoscopic target. ${ }^{13}$

Despite endoscopically normal appearing mucosa, it is recognised that a sizeable proportion of patients with UC will continue to have histologic activity. ${ }^{14-21}$ There are several studies that have examined whether persistent histologic changes modify long-term prognosis ${ }^{15,17,22,23}$ but such studies have several limitations that preclude robust interpretation of findings. First, many prior studies included patients with an endoscopic score of 0 or $1 .^{17,23}$ As histologic activity correlates with endoscopic severity, findings from such studies cannot be extrapolated to define the impact of histologic activity on prognosis of patients with completely normal mucosal appearance. Second, studies have retrospectively stratified histologic activity using various definitions and/or did not utilise a validated histologic activity score. ${ }^{17,19}$ Thus, whether histologic normalisation or persistence of inflammatory infiltrate modifies short- and medium-term outcomes in patients with UC with a completely normal endoscopic appearance has not been robustly established.

Therefore, in patients with UC with endoscopically normal mucosa (endoscopic subscore of 0 ), we aimed to examine if histologic normalisation or persistence of inflammatory activity quantified using a validated scale of histologic activity was associated with (a) risk of disease relapse, need for UC-related surgery or hospitalisation over the subsequent 2 years; and (b) concurrent symptoms related to UC.

\section{2 | METHODS}

\section{1 | Study cohort}

This was a single centre study based at a tertiary referral IBD centre serving the population of Boston and the surrounding New England area. To be eligible for inclusion, patients were required to meet the following criteria: (a) established diagnosis of UC according to standard diagnosis criteria; (b) enrolment in the prospective institutional registry; (c) a colonoscopy with complete endoscopic remission defined as a Baron score of 0 (normal mucosa with no erythema or friability) ${ }^{24}$; and (d) clinical follow-up of 2 years. Of all eligible patients, four were excluded (two with no follow-up information and two with a caecal patch or mild right colonic activity). We did not require clinical remission as recent studies have demonstrated that persistent alteration in bowel frequency may remain even with endoscopic and histologic remission and is likely from non-inflammatory changes. ${ }^{25}$

\subsection{Clinical covariates and outcome of interest}

The primary outcome was clinical relapse within two years from the time of the index colonoscopy. This was defined as any change in UC therapy (ie dose escalation, class change and/or need for systemic corticosteroids), UC-related hospitalisation or UC-related surgery within 2 years. Only dose change or medication alterations for symptomatic exacerbations of disease with clinical suspicion for active UC were characterised as relapse. Dose or therapy change for insurance-related reasons or patient preference was not considered relapse. Patients in whom the colonoscopy was performed explicitly with the intention of de-escalating or ceasing therapy were excluded. All charts were independently reviewed for the outcome of interest.

Additional covariates included in the study cohort included age at diagnosis, age at visit, disease duration, disease extent, medication use at the time of the study visit, clinical disease activity as measured using the simple clinical colitis activity index (SCCAI), ${ }^{26}$ anxiety and depression scores as measured using the Patient-Reported Outcomes Measurement Information System (PROMIS) questionnaires. Current immune modulator use was recorded for either thiopurine (azathioprine, 6-mercaptopurine) or methotrexate therapy. Current biologic use was recorded for either tumour necrosis factor $\alpha$ antagonists (infliximab, adalimumab, golimumab) or anti-integrin (vedolizumab) therapy.

\section{3 | Assessment of histologic activity}

All histologic specimens from the index colonoscopy were reviewed by a single senior gastrointestinal pathologist (VD) who was blinded to the outcome of interest. The site with the most histologically active disease was scored according the Geboes score. ${ }^{27}$ The individual components of the Geboes score were recorded for each patient: structural (architectural change) (Grade 0), chronic inflammatory infiltrate (Grade 1), lamina propria eosinophils (Grade 2A), lamina propria neutrophils (Grade 2B), neutrophils in the epithelium (Grade 3), crypt destruction (Grade 4) and erosion or ulceration (Grade 5). ${ }^{27}$ The expected lymphoplasmacytic infiltrate in the colonic segment chosen for review was accounted for when scoring the grade "chronic inflammatory infiltrate". We modelled histologic activity as a predictor variable using three definitions. ${ }^{28}$ First, patients were classified as having histologically active disease if the 
Geboes score was $\geq 3.1$. According to the second definition, histologically active disease was defined as Geboes score was $\geq 2.1$. Finally, histologic normalisation was defined as the complete absence of any histologic inflammatory activity, no increased chronic inflammatory infiltrate, no neutrophils and no structural and architectural change (Geboes score $=0$ ).

While the primary histologic assessment was using the Geboes score, we also examined the predictive value of the Robarts Histologic Index $(\mathrm{RHI})^{29}$ and the Nancy Index ${ }^{30}$ in determining risk of relapse. To do so, features from the Geboes score were extrapolated to the two other indices and total $\mathrm{RHI}$ and Nancy scores derived as previously defined. ${ }^{25}$ We compared the risk of relapse across different strata of the Nancy histologic score. For the RHI, we examined the predictive value of each of the separate cut-offs for histologic remission that have been proposed $\left(\leq 1,,^{31} \leq 3,{ }^{31}\right.$ and $\left.\leq 6^{32}\right)$ in addition to comparing the total $\mathrm{RHI}$ between relapsing and nonrelapsing patients.

\section{4 | Statistical analysis}

The study was approved by the Institutional Review Board of Partners Healthcare. All patients provided informed consent. Categorical variables are presented as counts and percentages. Comparisons between categorical variables were performed using the chi-squared test or Fisher exact test, as appropriate. Continuous variables are presented as means \pm standard deviations or medians \pm interquartile ranges based on normal distribution of the variable. Comparisons between continuous variables were performed with the student's $t$ test or Wilcoxon rank sum test, as appropriate. First, univariate regression was performed against the outcome of relapse to identify potentially influential factors. Covariates significant in this analysis at $P<0.05$ or those that had been previously determined to be important in predicting relapse were included in a final multivariable model that examined the independent effect of histologic changes. A two-sided $P<0.05$ indicated independent statistical significance. A receiver operating curve (ROC) was constructed for the final model and the area under the curve (AUC) was calculated. We repeated the analysis using Cox regression models and plotted a Kaplan-Meier curve of time to relapse stratifying by histologic normalisation or activity. Analysis were performed in R (version 3.5.3) using RStudio, version 1.1.456, and Stata 13.2 (StataCorp). ${ }^{33-35}$ The "yardstick" package was used for ROC construction and AUC calculation. ${ }^{36}$

\section{3 | RESULTS}

\subsection{Study cohort}

There were a total of 811 patients with UC in the prospective institutional registry. There was an equal distribution of men and women (50\% men, $50 \%$ women) with a median age of diagnosis of 27 years. Most patients had pancolitis (55\%) with $66 \%$ exposed to immune modulators and $39 \%$ exposed to anti-TNF therapy. The included study cohort was comparable to the full registry cohort in terms of study demographics and disease extent (26 years vs 27 years; $49 \%$ male vs $50 \%$ male; $55 \%$ with pancolitis vs $55 \%$ with pancolitis) but had less frequent use of immune modulators ( $41 \%$ vs $66 \%$ ) and antiTNFs (30\% vs $39 \%)$. In the final study cohort, there were a total of 83 participants with UC who met the specified inclusion criteria. The median disease duration was 11 years. About half the patients with complete endoscopic remission (endoscopic subscore of 0 ) demonstrated complete histologic normalisation by the Geboes score ( $n=41,49 \%$ ). A total of 20 patients (24\%) and 27 patients (33\%) met the criteria for histologically active disease defined using a Geboes score of $\geq 3.1$ and $\geq 2$.1a respectively. Interestingly, fewer patients on biologic therapy had histologically active disease, as defined by a Geboes score $\geq 3.1$ ( $11 \%$ vs $31 \%, P=0.08$ ); though this did not reach statistical significance.

Over a follow-up of 2 years, the primary study outcome (disease relapse) occurred in 26 patients (31\%). Among the patients who relapsed, one required surgery, three required hospitalisation for a flare of their UC, 16 were initiated on systemic steroids, 11 were started on topical therapy, four required new initiation of biologic therapy, three changed their biologic agent due to loss of response and two additionally had a dose increase of thiopurine. Only two patients de-escalated therapy after the index colonoscopy-one stopped mesalazine (mesalamine) and the other stopped anti-TNF. Both relapsed of which one had complete histologic normalisation at baseline while the other had histologically active disease. Exclusion of these two patients from the analysis did not alter our findings. Clinical symptoms, defined as an SCCAI $\geq 2$, were present in 19 of 81 patients assessed at the time of colonoscopy (23\%) despite complete endoscopic remission. Patients who experienced clinical relapse within two years of histologic evaluation were similar in demographic characteristics compared to those who did not relapse (Table 1). Age at diagnosis (27 vs 25 years, $P=0.78$ ), disease duration (14 vs 10 years, $P=0.11)$, gender $(P=0.34)$ and race/ethnicity $(P=0.59)$ were not significantly different between those who relapsed compared to those who did not. There was also no difference in current use of immune modulators $(P=1.00)$ or biologics $(P=0.52)$ between the two groups.

\section{2 | Histologic changes and relapse}

Patients with complete histologic normalisation by the Geboes score were less likely to experience relapse (12\%) compared to those without $(50 \%, P<0.001)$ (Figure 1). In contrast, histologic activity as assessed by a Geboes score of $\geq 2.1$ a ( $44 \%$ vs $25 \%$, $P=0.12), \geq 2.1 \mathrm{~b}(38 \%$ vs $29 \%, P=0.62)$ and $\geq 3.1$ (40\% vs $28 \%$, $P=0.49)$ was not significantly associated with risk of relapse (Table 2). However, the percentage of patients with clinical relapse was higher in all groups with higher Geboes scores (Figure 2). Two subcomponents of the Geboes score demonstrated significant associations with risk of clinical relapse within 2 years (Table 3). The strongest association was observed with the presence of increased numbers of chronic inflammatory cells $(P<0.001)$ with 
TAB LE 1 Characteristics of study participants based on relapse status within 2 years of index examination

\begin{tabular}{|c|c|c|c|}
\hline & $\begin{array}{l}\text { Clinical re- } \\
\text { lapse }(n=26)\end{array}$ & $\begin{array}{l}\text { No clinical re- } \\
\text { lapse }(n=57)\end{array}$ & $P$ value \\
\hline Age at diagnosis & $27.5 \pm 15.3$ & $25 \pm 20$ & 0.78 \\
\hline Age at visit & $44.5 \pm 19.3$ & $43 \pm 23$ & 0.57 \\
\hline Disease duration & $14.5 \pm 12$ & $10 \pm 9$ & 0.11 \\
\hline \multicolumn{4}{|l|}{ Gender } \\
\hline Male & $10(38.4 \%)$ & $30(52.6 \%)$ & 0.34 \\
\hline Female & $16(61.5 \%)$ & $27(47.4 \%)$ & \\
\hline \multicolumn{4}{|l|}{ Race } \\
\hline White & $24(92.3 \%)$ & $55(96.5 \%)$ & $0.59^{*}$ \\
\hline Non-white & $2(7.7 \%)$ & $2(3.5 \%)$ & \\
\hline \multicolumn{4}{|l|}{ Pancolitis } \\
\hline Yes & $14(53.8 \%)$ & $32(56.1 \%)$ & 1.00 \\
\hline No & $12(46.2 \%)$ & $25(43.9 \%)$ & \\
\hline \multicolumn{4}{|c|}{ Current immune modulator } \\
\hline Yes & $11(42.3 \%)$ & $23(40.4 \%)$ & 1.00 \\
\hline No & $15(57.7 \%)$ & $34(59.6 \%)$ & \\
\hline \multicolumn{4}{|l|}{ Current biologic } \\
\hline Yes & $7(26.9 \%)$ & $21(36.8 \%)$ & 0.52 \\
\hline No & 19 (73.1\%) & $36(63.2 \%)$ & \\
\hline \multicolumn{4}{|l|}{ Current steroid } \\
\hline Yes & $2(7.7 \%)$ & $2(3.5 \%)$ & $0.59^{*}$ \\
\hline No & 24 (92.3\%) & 55 (96.5\%) & \\
\hline
\end{tabular}

${ }^{*}$ Fisher's exact test.

more patients who had clinical relapse having a mild (65\% vs $23 \%$ ) or moderate increase ( $15 \%$ vs $5 \%$ ). Most patients with no clinical relapse within two years had no increase in chronic inflammatory infiltrate (65\%). The second significant association with clinical relapse occurred with the presence of architectural changes $(P=0.03)$. More patients who experienced clinical relapse within 2 years had a mild abnormality ( $31 \%$ vs $10 \%$ ) or mild-moderate diffuse or multifocal abnormalities ( $8 \%$ vs $3 \%$ ). Most patients in the group who did not experience clinical relapse within two years had no abnormality (86\%). The presence of lamina propria neutrophils $(P=0.69)$ and/or eosinophils $(P=0.08)$ was not statistically significant between those who relapsed and those who did not. There was also no difference between groups in the presence of neutrophils in the epithelium $(P=0.35)$ or crypt destruction $(P=0.59)$. A Kaplan-Meier survival curve describing time to relapse similarly demonstrated a lower frequency of, and longer time to, relapse among those achieving complete histologic normalisation (logrank $P=0.0002$ ) but not when stratifying by histologic activity at a cut-off of Geboes $3.1(P=0.52)$ (Figure 3).

\section{3 | Multivariable predictors of relapse}

Lack of complete histologic normalisation by the Geboes score was predictive of clinical relapse at two years (OR $7.22,95 \% \mathrm{Cl}$ 2.48-24.70) when controlling for disease duration, current biologic use and age at time of the visit (Table 4). Age at visit (OR 0.99, 95\% $\mathrm{Cl}$ 0.96-1.04), disease duration (OR 1.03, 95\% Cl 0.97-1.08) and biologic use (OR $0.75,95 \% \mathrm{Cl} 0.24-2.28$ ) were not predictive of clinical relapse within two years. The ROC curve demonstrated good predictive ability of the Geboes score to discriminate the outcome of interest, clinical relapse, with an AUC of 0.78 (Figure 4).

\subsection{Predictive models using the Robarts Histologic Index and Nancy Scores}

We examined whether the RHI or Nancy score while in endoscopic remission predicted relapse. The distribution of the Nancy index in the cohort is presented in Table S1. There was no statistically significant difference in rates of relapse across the Nancy histologic scores, with relapse rates ranging from $25 \%$ (two out of eight patients) with a Nancy score of 3 and $50 \%$ (one out of two patients) with a Nancy score of 1 . There were no patients with a Nancy score of 4 . The rates of relapse in those with a Nancy score of 0 (28\%) was similar to those with a score $\geq 1$ (39\%, $P=0.34)$. The RHI in our cohort ranged from 0 to 13 (median 1, IQR 0-4). The mean

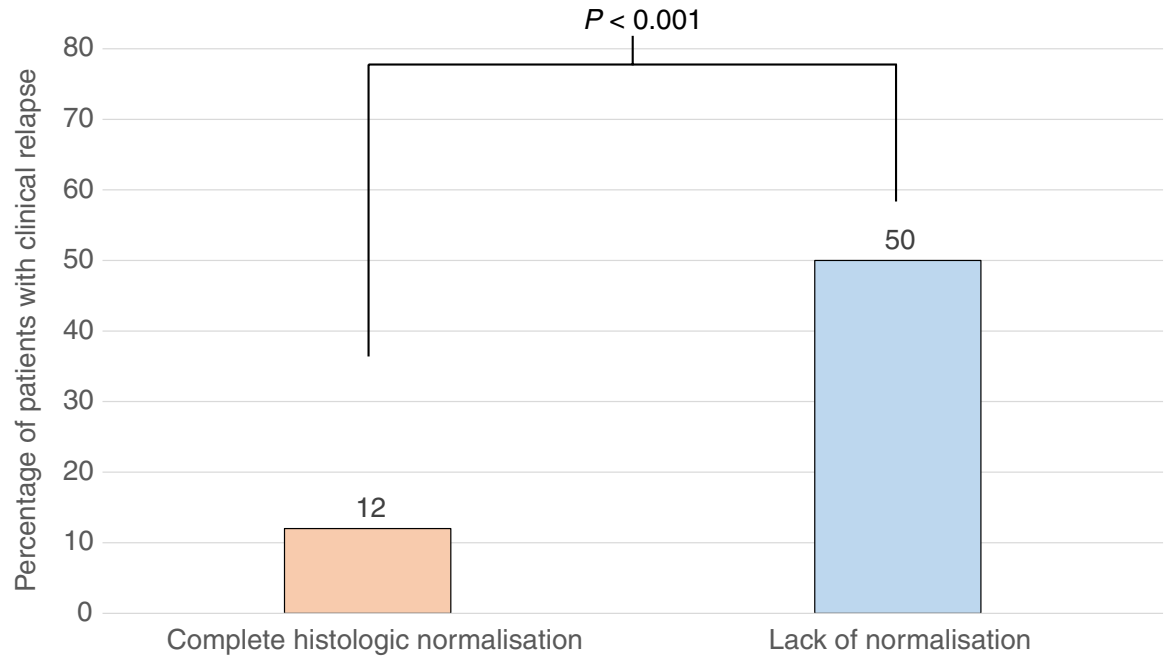

FIGURE 1 Percentage of patients with clinical relapse within 2 years of endoscopy by histologic normalisation (Geboes score 0:5/41, 12\%, Geboes Score $>0$ : (21/42, 50\%). Clinical relapse: defined as need for therapy escalation, surgery and/or hospitalisation 
TAB LE 2 Association between histologic activity and clinical relapse, by Geboes score classification

\begin{tabular}{|c|c|c|c|}
\hline & $\begin{array}{l}\text { Clinical relapse } \\
(n=26)\end{array}$ & $\begin{array}{l}\text { No clinical relapse } \\
(n=57)\end{array}$ & $P$ value \\
\hline \multicolumn{4}{|c|}{ Lack of normalisation (Geboes > 0) } \\
\hline Yes & $21(50 \%)$ & $21(50 \%)$ & $<0.001$ \\
\hline No & $5(12 \%)$ & $36(88 \%)$ & \\
\hline \multicolumn{4}{|c|}{ Geboes $\geq 2.1 \mathrm{a}$} \\
\hline Yes & $12(44 \%)$ & $15(56 \%)$ & 0.12 \\
\hline No & $14(25 \%)$ & $42(75 \%)$ & \\
\hline \multicolumn{4}{|c|}{ Geboes $\geq 2.1 b$} \\
\hline Yes & $8(38 \%)$ & $13(62 \%)$ & 0.62 \\
\hline No & $18(29 \%)$ & $44(71 \%)$ & \\
\hline \multicolumn{4}{|c|}{ Geboes $\geq 3.1$} \\
\hline Yes & $8(40 \%)$ & $12(60 \%)$ & 0.49 \\
\hline No & $18(28 \%)$ & 45 (72\%) & \\
\hline
\end{tabular}

$\mathrm{RHI}$ in patients who relapsed $(2.23 \pm 2.57)$ was similar to those who did not relapse $(1.72 \pm 3.18)(P=0.47)$. Dichotomising histologic remission using the cut-offs of $\leq 1^{31}$ (39\% vs $28 \%, P=0.34$ ), $\leq 3^{31}$ (38\% vs $\left.29 \%, P=0.44\right)$, and $\leq 6^{32}(25 \%$ vs $32 \%, P=0.69)$ did not reveal any significant associations between $\mathrm{RHI}$ and risk of relapse.

\subsection{Association between histologic changes and disease symptoms}

Sixty-two patients (77\%) were in clinical remission. No difference was observed in total SCCAI score between those who had histologic activity as defined by a Geboes score $\geq 3.1(P=0.17)$ or lack of complete histologic normalisation $(P=0.60)$. There was also no difference in individual symptoms by histologic activity. Specifically, when histologic activity was defined as a Geboes score of $\geq 3.1$, there was no difference in faecal urgency $(20 \%$ vs $35 \%, P=0.27)$, general well-being ( $15 \%$ vs $27 \%, P=0.37$ ), blood in the stool $(15 \%$ vs $8 \%, P=0.39$ ), daily bowel frequency ( $0 \%$ vs $10 \%, P=-0.33$ ) or nocturnal bowel frequency ( $0 \%$ vs $2 \%, P=1.00$ ) between the two groups (Table S2). Similarly, there was no difference in any of these symptoms between those who had complete histologic normalisation and those who did not (Table S3).

\section{4 | DISCUSSION}

The treatment target in UC has evolved from symptomatic remission to endoscopic remission with the recognition that persistent endoscopic inflammatory activity even in the absence of symptoms is associated with worse long-term outcomes. ${ }^{4-6}$ However, the recognition that histologic activity persists even in a significant subset of patients with complete endoscopic normalisation has led to consideration of histologic healing as the optimal therapeutic target. ${ }^{4-6}$ However, studies of histologic activity have been either cross-sectional in examining associations with symptoms, ${ }^{25}$ used nonvalidated retrospective assessments of histologic activity ${ }^{17}$ or did not examine longer-term outcomes. Further, few studies have attempted to define the optimal histologic target necessary for modifying future disease course and whether this differs from that used for assessing therapeutic response to pharmacologic intervention. ${ }^{37,38}$ In this study, using blinded expert histologic assessments in patients with UC in complete endoscopic remission, we demonstrate that complete histologic normalisation with absence of structural/ architectural features rather than resolution of active inflammatory infiltrate was associated with reduced future risk of relapse. These results may suggest incremental benefit in attaining histologic remission in UC and further suggest that features that discriminate therapeutic effect may be different compared to those required to alter disease course. However, attainment of histologic remission is still infrequent with the existing therapies, and while our study may provide prognostic information
FIGURE 2 Percentage of patients who have clinical relapse by Geboes score threshold within 2 years of endoscopy

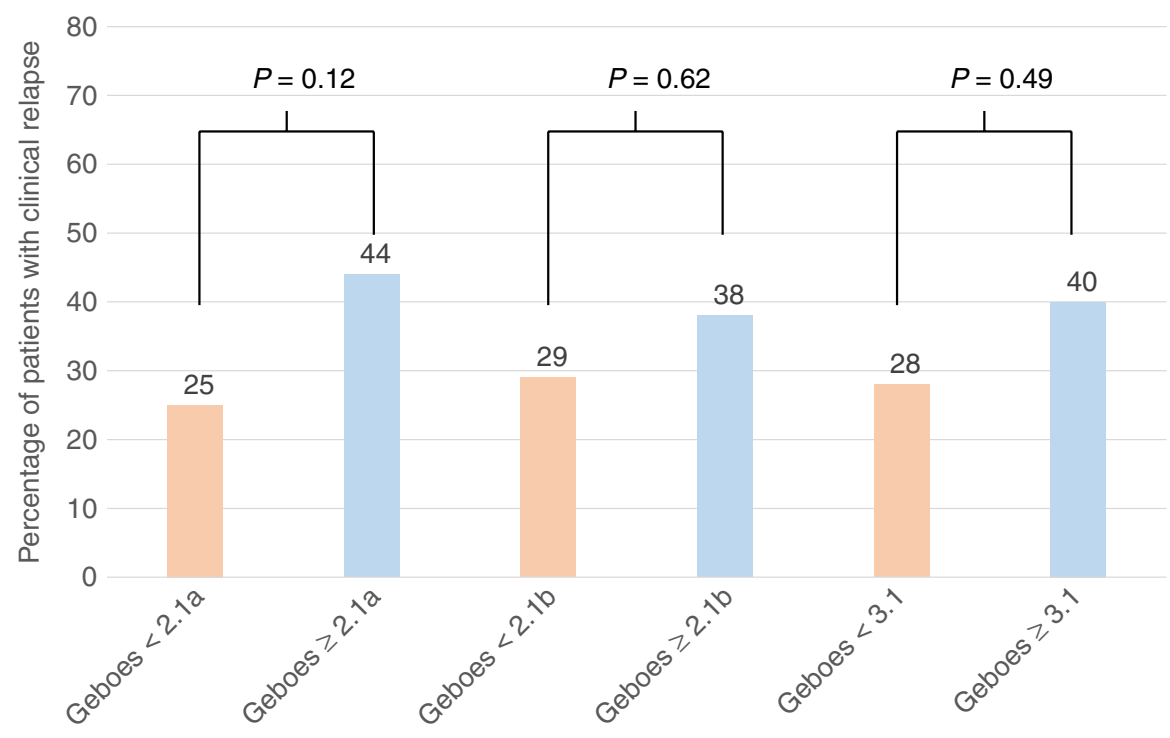


TAB LE 3 Components of the Geboes score by clinical relapse within 2 years of colonoscopic evaluation among patients with ulcerative colitis

\begin{tabular}{|llll|}
\hline & $\begin{array}{l}\text { Clinical } \\
\text { relapse } \\
(\mathbf{n = 2 6 )}\end{array}$ & $\begin{array}{l}\text { No clinical } \\
\text { relapse } \\
(\mathbf{n = 5 7 )}\end{array}$ & P value \\
\hline $\begin{array}{clll}\text { Structural (architectural change) } \\
\text { No abnormality }\end{array}$ & $16(61.5 \%)$ & $49(86 \%)$ & 0.03 \\
\hline $\begin{array}{c}\text { Mild Abnormality } \\
\text { Mild or moderate diffuse or } \\
\text { multifocal abnormalities }\end{array}$ & $8(30.8 \%)$ & $6(10.5 \%)$ & \\
\hline $\begin{array}{c}\text { Severe diffuse or multifocal } \\
\text { abnormalities }\end{array}$ & 0 & $2(3.5 \%)$ & \\
\hline $\begin{array}{c}\text { Chronic inflammatory infiltrate } \\
\text { No increase }\end{array}$ & $5(19.2 \%)$ & $37(64.9 \%)$ & \\
\hline Mild but unequivocal increase & $17(65.4 \%)$ & $13(22.8 \%)$ & \\
\hline Moderate increase & $4(15.4 \%)$ & $3(5.3 \%)$ & \\
\hline Marked increase & 0 & $4(7 \%)$ & \\
\hline
\end{tabular}

Lamina Propria neutrophils and eosinophils

2 A: Eosinophils

No increase

Mild but unequivocal increase

Moderate increase

Marked increase

2B: Neutrophils

$\begin{array}{lll}\text { None } & 26(100 \%) & 53(93 \%) \\ \begin{array}{l}\text { Mild but unequivocal } \\ \text { increase }\end{array} & 0 & 3(5.3 \%) \\ \text { Moderate increase } & 0 & 1(1.7 \%) \\ \text { Marked increase } & 0 & 0\end{array}$

Neutrophils in the epithelium

None

$<5 \%$ crypts involved

$<50 \%$ crypts involved

$>50 \%$ crypts involved

$16(61.5 \%)$

$7(26.9 \%)$

$1(3.8 \%) \quad 0$

$2(7.7 \%)$

$1(1.8 \%)$

$0.69^{*}$

Crypt destruction

$\begin{array}{lll}\text { None } & 24(92.3 \%) & 55(96.5 \%) \\ \begin{array}{l}\text { Probable-local excess of } \\ \text { neutrophils in part of crypt }\end{array} & 2(7.7 \%) & 2(3.5 \%) \\ \text { Probably-marked attenuation } & 0 & 0 \\ \begin{array}{l}\text { Unequivocal crypt } \\ \text { destruction }\end{array} & 0 & 0\end{array}$
destruction

Erosion or Ulceration

N/A

No erosion, ulceration, or $\quad 26(100 \%) \quad 57(100 \%)$ granulation tissue

$\begin{array}{lll}\begin{array}{c}\text { Recovering epithelium }+ \\ \text { adjacent inflammation }\end{array} & 0 & 0 \\ \begin{array}{l}\text { Probable erosion-focally } \\ \text { stripped }\end{array} & 0 & 0\end{array}$

(Continues)
TABLE 3 (Continued)

\begin{tabular}{llll} 
& $\begin{array}{lll}\text { Clinical } \\
\text { relapse } \\
(n=26)\end{array}$ & $\begin{array}{l}\text { No clinical } \\
\text { relapse } \\
(n=57)\end{array} \quad$ P value \\
\hline Unequivocal erosion & 0 & 0 & \\
\hline Ulcer or granulation tissue & 0 & 0 & \\
\hline
\end{tabular}

${ }^{*}$ Fisher's Exact test.

to clinicians when faced with a discrepancy between histologic activity and endoscopic remission, there are still insufficient data to recommend histologic healing as a necessary therapeutic target in all patients.

A few prior studies have evaluated the impact of histologic disease activity on clinical outcomes in UC. A large retrospective cohort by Christensen et al demonstrated that histologic normalisation was beneficial even in those with endoscopic remission, but histologic activity was not assessed using validated scoring systems. ${ }^{17}$ Of those that have used histologic scores, most have relied on the Geboes score. Several have used a Geboes score with a threshold of $\geq 3.1$ to define histologic activity. ${ }^{15,21,39,40}$ Bessissow et al retrospectively evaluated the role of histologic disease activity on clinical relapse within 12 months among 75 patients with UC who had Mayo 0 endoscopic disease. ${ }^{15}$ The authors found that a Geboes score $\geq 3.1$ and basal plasmacytosis were associated with relapse on univariate analysis but only basal plasmacytosis was associated with relapse on multivariate analysis. Zenlea et al evaluated the role of histology as a predictor of clinical relapse in UC among patients who were in clinical remission. ${ }^{21}$ The authors found that Geboes score $\geq 3.1$ was associated with clinical relapse on both univariate and multivariate analysis. Our study expands upon previous findings by showing that histologic activity was associated with clinical relapse among a larger group of patients in complete endoscopic remission and expands the histological analysis to include derived $\mathrm{RHI}$ and Nancy histology indices.

A systematic review and meta-analysis performed in 2016 investigated the available data in this area to determine the association between histologic healing and relapse. ${ }^{14}$ The authors found that histologic remission was associated with reduced risk of relapse (relative risk 0.48). Furthermore, absence of neutrophils in the epithelium, neutrophils or eosinophils in the lamina propria, crypt abscesses and chronic inflammatory cell infiltrates were all associated with lower risk of clinical relapse. One explanation of the heterogeneity in previous findings including association between histologic remission and relapse in prior studies is inclusion of patients with endoscopic score 0 or 1 . However, one limitation of this analysis is pooling together of patients across a spectrum of endoscopic activity. Patients with an endoscopic subscore of 1 are more likely to have histologic disease activity and also more likely to relapse, raising the possibility of confounding in the prior studies. With increasing acceptance of endoscopic subscore 0 as the treatment target for endoscopic remission and strong correlation between histologic and endoscopic activity, it is important to robustly examine the additive benefit of histologic inflammation or 

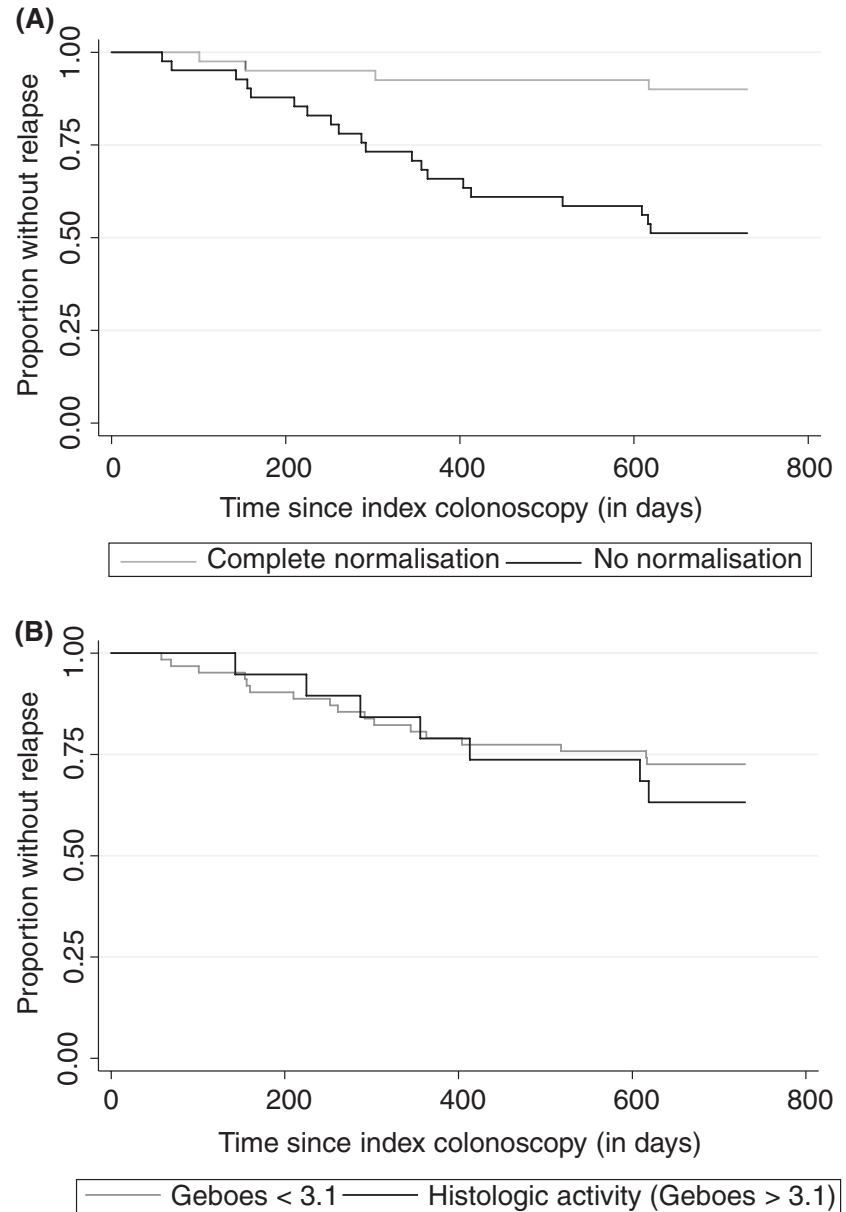

FIGURE 3 Kaplan-Meier curve of time to relapse in patients with ulcerative colitis in endoscopic remission stratified by (A) complete histologic normalisation (Geboes score = 0; $n=41$ ); and (B) histologic activity (Geboes score $\geq 3.1 ; \mathrm{n}=20$ )

normalisation in this subpopulation rather than in a more heterogenous group of patients with broadly varying endoscopic activity. In our study, larger than these prior two and with a longer duration of follow up, we did not detect an association between histologically active disease and clinical relapse using various Geboes thresholds, the RHI, or Nancy index. However, interestingly, resolution of chronic architectural distortion was one of the two features in the Geboes score that was most predictive of relapse and this characteristic is not a part of the RHI or Nancy index. This finding intriguingly suggests that perhaps histologic changes that modify disease course (like relapse) are different from those that can measure therapeutic response to an intervention (like RHI or Nancy score). This merits further examination in future prospective studies.

There are several strengths of this study. First, all patients had extensive data available for review given that they were followed as part of a prospective registry. This allowed us to examine associations between symptoms at the time of the endoscopy and biopsy collection as well as have up to date information on current medication use at the time of endoscopy. Second, histologic specimens were reviewed by a senior gastrointestinal pathologist who was blinded to the outcome of interest. This limited the potential
TAB LE 4 Multivariable logistic regression evaluating predictors of clinical relapse at 2 years

\begin{tabular}{|llll} 
& Odds ratio & $95 \% \mathrm{Cl}$ & P value \\
\hline $\begin{array}{l}\text { Lack of complete } \\
\text { normalisation }\end{array}$ & 7.22 & $2.48-24.7$ & $<0.001$ \\
\hline Disease duration & 1.03 & $0.97-1.08$ & 0.38 \\
\hline Current biologic use & 0.75 & $0.24-2.28$ & 0.68 \\
\hline Age at visit & 0.99 & $0.96-1.04$ & 0.93 \\
\hline
\end{tabular}

${ }^{a}$ Complete histologic normalisation indicates a Geboes score of 0 .

for observer bias. Third, we used the Geboes score for primary histologic assessment but also included RHI and Robarts. To our knowledge, ours is the first study to simultaneously examine all three scoring systems to predict relapse. The Geboes score is a comprehensive histologic scoring system which evaluates both acute and chronic inflammatory changes in addition to architectural distortion. This score has been shown to have good concordance with other histologic scoring systems in inflammatory bowel disease including the Robarts score and the Nancy Index. ${ }^{28}$ Further, the comprehensiveness of the Geboes score allowed us to evaluate several thresholds to score histologic activity as well as transpose to derive $\mathrm{RHI}$ and Nancy histology indices. Finally, we isolated the impact of histologic activity on the outcome of clinical relapse by defining endoscopic remission using a strict threshold, which was a Baron score of 0 . By excluding a Baron score of 1 , we could evaluate an extremely well-controlled group of patients and isolate the impact of histology on relapse in this group.

We readily acknowledge some limitations of this work. First, there was a relatively small sample size which may have limited

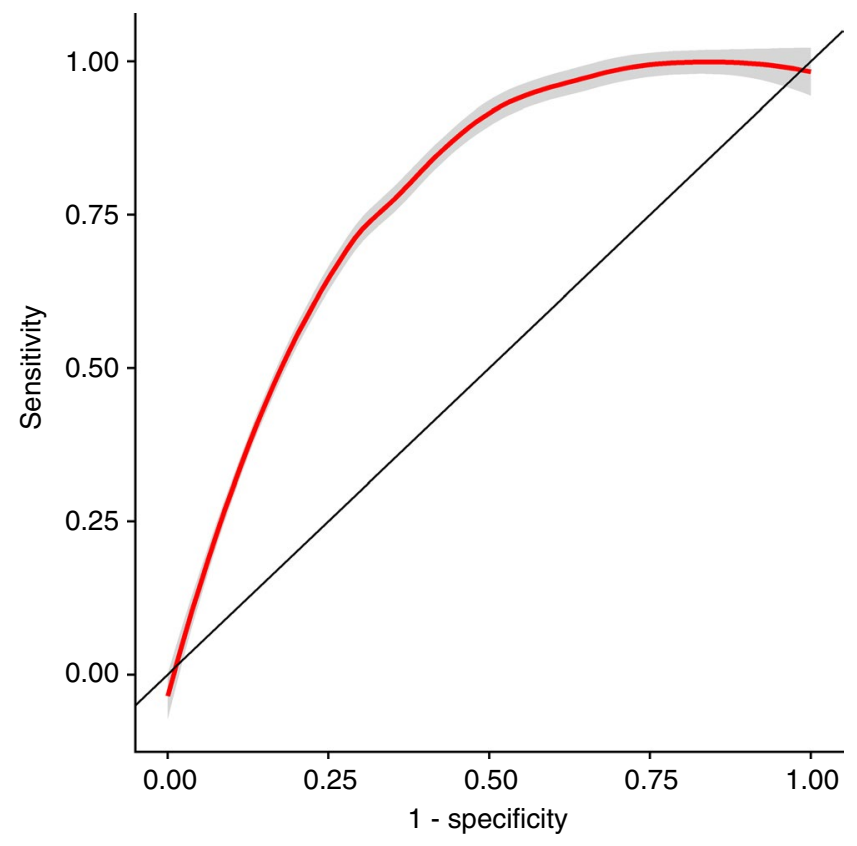

FIGURE 4 Accuracy of histologic normalisation in predicting clinical relapse in patients with ulcerative colitis in endoscopic remission (area under the curve $=0.78$ ) 
power for statistical analyses. Despite this, ours remains one of the largest to examine the value of histologic activity on medium-term natural history in patients with UC in complete endoscopic remission. We did not have sufficient numbers to examine the predictive value of histology within subgroups of patients by disease extent or existing treatments. One such consideration would be the influence of steroids prior to colonoscopy which could bias towards increased histologic healing. However, if there were transient steroid exposure (due to a recent flare) resulting in histologic healing, this would be expected to bias the population with healing towards a higher rate of relapse in the future (and lack of significance between groups), which we did not see. Second, histologic assessment was performed by one expert gastrointestinal pathologist. A recent study by Romkens, et al highlighted a concern for reproducibility in histologic scoring but this was most notable between general and expert gastrointestinal pathologists, which was not the case for this study which utilised expert review only. ${ }^{41}$ However, future studies would benefit from incorporation of more than one expert GI pathologist to arrive at consensus histologic assessments as well as repeat assessments by an individual pathologist to look at intra-rater consistency in scoring. Third, these data showed no association between active inflammatory infiltrate and subsequent risk of relapse which has been shown in previous studies. We hypothesise that this was most likely secondary to the small numbers of those with active inflammatory infiltrate at the time of colonoscopy (less than a third of the patient cohort) limiting statistical power. Fourth, we used clinically meaningful endpoints including relapse, hospitalisation and surgery. However, structured disease activity assessments using validated scores were not available systematically following the index examination. Future studies should be prospective with a systematic algorithm for evaluation and confirmation of symptomatic flares. Overall, the small sample size, small event rate in the Geboes 0 group and retrospective nature of the design are important limitations of this study. However, this work offers the most comprehensive evaluation of the association between histologic healing and medium-term ulcerative colitis outcomes to date.

In conclusion, histologic normalisation, but not resolution of inflammatory infiltrate, was associated with a reduced risk of clinical relapse within two years of endoscopic remission among patients with ulcerative colitis. The value of histologic remission as a therapeutic target in IBD continues to evolve. While mucosal and clinical remission remain as dual endpoints in therapeutic decision-making, the ability to achieve deeper remission with complete histologic healing may not be achievable in all patients. ${ }^{37,42,43}$ Our findings highlight the importance of consensus definitions across studies regarding the definition of histologic remission and establishment of relevant cut-offs not just for therapeutic response but also to modify long-term history of disease to optimise patient outcomes.

\section{ACKNOWLEDGEMENTS}

Declaration of personal interests: Kelly Cushing and William Tan have no conflict of interests to declare. David Alpers: Consulting for Pfizer, GSK, Otsuka North America, Takeda North America. Vikram Deshpande: Consulting for Agios; research support ACD. Ashwin Ananthakrishnan has served on scientific advisory boards for Abbvie, Gilead, Takeda and Merck.

\section{AUTHORSHIP}

Guarantor of the article: Ashwin N Ananthakrishnan.

Author contributions: Cushing was involved in the study design and data analysis in addition to writing the manuscript, reviewing the manuscript and approving the final version of the manuscript. Tan was involved in data collection in addition to reviewing the manuscript and approving the final version of the manuscript. Alpers was involved in the study design in addition to reviewing the manuscript and approving the final version of the manuscript. Deshpande was involved in the study design and data collection in addition to reviewing the manuscript and approving the final version of the manuscript. Ananthakrishnan was involved in the study design and data analysis in addition to writing the manuscript, reviewing the manuscript and approving the final version of the manuscript.

All authors approved the final version of the manuscript.

\section{ORCID}

Kelly C. Cushing iD https://orcid.org/0000-0003-3914-9673

\section{LINKED CONTENT}

This article is linked to Pai et al and Cushing et al and Ananthakrishnan papers. To view these articles, visit https://doi.org/10.1111/ apt.15617 and https://doi.org/10.1111/apt.15621.

\section{REFERENCES}

1. Shivashankar R, Tremaine WJ, Harmsen WS, Loftus EV Jr. Incidence and prevalence of Crohn's disease and ulcerative colitis in Olmsted county, Minnesota from 1970 through 2010. Clin Gastroenterol Hepatol. 2017;15:857-863.

2. Ungaro R, Mehandru S, Allen PB, Peyrin-Biroulet L, Colombel JF. Ulcerative colitis. Lancet. 2017;389:1756-1770.

3. Agrawal M, Colombel JF. Treat-to-target in inflammatory bowel diseases, what is the target and how do we treat? Gastrointest Endosc Clin N Am. 2019;29:421-436.

4. Peyrin-Biroulet L, Sandborn W, Sands BE, et al. Selecting therapeutic targets in inflammatory bowel disease (STRIDE): determining therapeutic goals for treat-to-target. Am J Gastroenterol. 2015;110:1324-1338.

5. Ungaro R, Colombel JF, Lissoos T, Peyrin-Biroulet L. A treatto-target update in ulcerative colitis: a systematic review. Am J Gastroenterol. 2019;114:874-883.

6. Pineton de Chambrun G, Blanc P, Peyrin-Biroulet L. Current evidence supporting mucosal healing and deep remission as important treatment goals for inflammatory bowel disease. Expert Rev Gastroenterol Hepatol. 2016;10:915-927.

7. Colombel JF, Rutgeerts $\mathrm{P}$, Reinisch W, et al. Early mucosal healing with infliximab is associated with improved long-term clinical outcomes in ulcerative colitis. Gastroenterology. 2011;141:11941201 
8. Ardizzone S, Cassinotti A, Duca P, et al. Mucosal healing predicts late outcomes after the first course of corticosteroids for newly diagnosed ulcerative colitis. Clin Gastroenterol Hepatol. 2011;9:483-489.e483.

9. Flores BM, O'Connor A, Moss AC. Impact of mucosal inflammation on risk of colorectal neoplasia in patients with ulcerative colitis: a systematic review and meta-analysis. Gastrointest Endosc. 2017;86:1006-1011.e8.

10. Meucci G, Fasoli R, Saibeni S, et al. Prognostic significance of endoscopic remission in patients with active ulcerative colitis treated with oral and topical mesalazine: a prospective, multicenter study. Inflamm Bowel Dis. 2012;18:1006-1010.

11. Barreiro-de Acosta M, Vallejo N, de la Iglesia D, et al. Evaluation of the risk of relapse in ulcerative colitis according to the degree of mucosal healing (mayo 0 vs 1): a longitudinal cohort study. J Crohns Colitis. 2016;10:13-19.

12. Manginot C, Baumann C, Peyrin-Biroulet L. An endoscopic Mayo score of 0 is associated with a lower risk of colectomy than a score of 1 in ulcerative colitis. Gut. 2015;64(7):1181-1182.

13. Fukuda T, Naganuma M, Sugimoto $S$, et al. Efficacy of therapeutic intervention for patients with an ulcerative colitis mayo endoscopic score of 1. Inflamm Bowel Dis. 2019;25:782-788.

14. Park S, Abdi T, Gentry M, Laine L. Histological disease activity as a predictor of clinical relapse among patients with ulcerative colitis: systematic review and meta-analysis. Am J Gastroenterol. 2016;111:1692-1701.

15. Bessissow $T$, Lemmens $B$, Ferrante $M$, et al. Prognostic value of serologic and histologic markers on clinical relapse in ulcerative colitis patients with mucosal healing. Am J Gastroenterol. 2012;107:1684-1692.

16. Calafat M, Lobatón T, Hernández-Gallego A, et al. Acute histological inflammatory activity is associated with clinical relapse in patients with ulcerative colitis in clinical and endoscopic remission. Dig Liver Dis. 2017;49:1327-1331.

17. Christensen B, Hanauer SB, Erlich J, et al. Histologic normalization occurs in ulcerative colitis and is associated with improved clinical outcomes. Clin Gastroenterol Hepatol. 2017;15:1557-1564.e1.

18. Patil DT, Moss AC, Odze RD. Role of histologic inflammation in the natural history of ulcerative colitis. Gastrointest Endosc Clin N Am. 2016;26:629-640.

19. Rosenberg L, Nanda KS, Zenlea T, et al. Histologic markers of inflammation in patients with ulcerative colitis in clinical remission. Clin Gastroenterol Hepatol. 2013;11:991-996.

20. Salem MS, Melmed GY. The role of histology in determining disease activity, treatment, and prognosis: are we there yet? Gastrointest Endosc Clin N Am. 2019;29:437-446.

21. Zenlea T, Yee EU, Rosenberg L, et al. Histology grade is independently associated with relapse risk in patients with ulcerative colitis in clinical remission: a prospective study. Am J Gastroenterol. 2016;111:685-690.

22. Riley SA, Mani V, Goodman MJ, DuttS, Herd ME. Microscopic activity in ulcerative colitis: what does it mean? Gut. 1991;32:174-178.

23. Ozaki R, Kobayashi T, Okabayashi S, et al. Histological risk factors to predict clinical relapse in ulcerative colitis with endoscopically normal mucosa. J Crohns Colitis. 2018;12:1288-1294.

24. Baron JH, Connell AM, Lennard-Jones JE. Variation between observers in describing mucosal appearances in proctocolitis. BMJ. 1964;1:89-92.

25. Colombel J-F, Keir ME, Scherl A, et al. Discrepancies between patient-reported outcomes, and endoscopic and histological appearance in UC. Gut. 2017;66:2063-2068.

26. Walmsley RS, Ayres RC, Pounder RE, Allan RN. A simple clinical colitis activity index. Gut. 1998;43:29-32.

27. Geboes K, Riddell R, Ost A, Jensfelt B, Persson T, Lofberg R. A reproducible grading scale for histological assessment of inflammation in ulcerative colitis. Gut. 2000;47:404-409.
28. Jairath V, Peyrin-Biroulet L, Zou G, et al. Responsiveness of histological disease activity indices in ulcerative colitis: a post hoc analysis using data from the TOUCHSTONE randomised controlled trial. Gut. 2019;68:1162-1168.

29. Mosli MH, Feagan BG, Zou G, et al. Development and validation of a histological index for UC. Gut. 2017;66:50-58.

30. Marchal-Bressenot A, Salleron J, Boulagnon-Rombi C, et al. Development and validation of the Nancy histological index for UC. Gut. 2017;66:43-49.

31. Pai RK, Khanna R, D'Haens GR, et al. Definitions of response and remission for the Robarts Histopathology Index. Gut. 2018;68:2101-2102.

32. Magro F, Lopes J, Borralho P, et al. Comparison of different histological indexes in the assessment of UC activity and their accuracy regarding endoscopic outcomes and faecal calprotectin levels. Gut. 2019;68(4):594-603.

33. RStudio Team. RStudio: Integrated Development for R. Boston, MA: RStudio I; 2016. https://www.rstudio.com/

34. StataCorp. Stata Statistical Software: Release 13. College Station, TX: StataCorp LP; 2013.

35. R Core Team. R: A Language and Environment for Statistical Computing. Vienna, Austria: R Foundation for Statistical Computing; 2018.

36. Vaughan MKaD. yardstick: Tidy Characterizations of Model Performance. 2019.

37. Battat R, Duijvestein M, Guizzetti L, et al. Histologic healing rates of medical therapies for ulcerative colitis: a systematic review and meta-analysis of randomized controlled trials. Am J Gastroenterol. 2019;114:733-745.

38. Ma C, Guizzetti L, Panaccione R, et al. Systematic review with meta-analysis: endoscopic and histologic placebo rates in induction and maintenance trials of ulcerative colitis. Aliment Pharmacol Ther. 2018;47:1578-1596.

39. Li CQ, Liu J, Ji R, Li Z, Xie XJ, Li YQ. Use of confocal laser endomicroscopy to predict relapse of ulcerative colitis. BMC Gastroenterol. 2014;14:45.

40. Zittan E, Kelly OB, Kirsch R, et al. Low fecal calprotectin correlates with histological remission and mucosal healing in ulcerative colitis and colonic Crohn's disease. Inflamm Bowel Dis. 2016;22: 623-630.

41. Römkens $T$, Kranenburg P, Tilburg AV, et al. Assessment of histological remission in ulcerative colitis: discrepancies between daily practice and expert opinion. J Crohns Colitis. 2018;12:425-431.

42. Long MD, Rubin DT. Histologic remission in ulcerative colitis: are we there yet? Am J Gastroenterol. 2019;114:713-715.

43. Sands BE, Peyrin-Biroulet L, Loftus EV, et al. Vedolizumab shows superior efficacy versus adalimumab: results of varsity: the first head-to-head study of biologic therapy for moderate-to-severe ulcerative colitis. Gastroenterology. 2019;156:S-81.

\section{SUPPORTING INFORMATION}

Additional supporting information will be found online in the Supporting Information section.

How to cite this article: Cushing KC, Tan W, Alpers DH, Deshpande V, Ananthakrishnan AN. Complete histologic normalisation is associated with reduced risk of relapse among patients with ulcerative colitis in complete endoscopic remission. Aliment Pharmacol Ther. 2020;51:347-355. https:// doi.org/10.1111/apt.15568 